\title{
Michel decay spectrum for a muon bound to a nucleus
}

\author{
Andrzej Czarnecki, ${ }^{1}$ Matthew Dowling, ${ }^{1, *}$ Xavier Garcia i Tormo, ${ }^{1,} \dagger$ William J. Marciano, ${ }^{2}$ and Robert Szafron ${ }^{1}$ \\ ${ }^{1}$ Department of Physics, University of Alberta, Edmonton, Alberta, Canada T6G $2 G^{7}$ \\ ${ }^{2}$ Department of Physics, Brookhaven National Laboratory, Upton, New York 11973, USA
}

\begin{abstract}
The spectrum of electrons from muons decaying in an atomic bound state is significantly modified by their interaction with the nucleus. Somewhat unexpectedly, its first measurement, at the Canadian laboratory TRIUMF, differed from basic theory. We show, using a combination of techniques developed in atomic, nuclear, and high-energy physics, that radiative corrections eliminate the discrepancy. In addition to solving that outstanding problem, our more precise predictions are potentially useful for interpreting future high-statistics muon experiments that aim to search for exotic interactions at $10^{-16}$ sensitivity.
\end{abstract}

PACS numbers: $13.35 . \mathrm{Bv}, 36.10 . \mathrm{Ee}$

Muons are very special elementary particles. They exhibit essentially the same electroweak interactions as electrons; however, their much larger mass $\left(m_{\mu} \simeq 207 m_{e}\right)$ endows them with some important features. Most noteworthy is the free muon decay rate which stems from its decay mode $\mu \rightarrow e \bar{\nu}_{e} \nu_{\mu}$. The differential decay rate as a function of the electron energy $[1,2]$ (neglecting $m_{e}^{2} / m_{\mu}^{2}$ and $\mathcal{O}\left(\alpha^{2}\right)$ effects [3]) is given by

$$
\begin{aligned}
& \frac{\mathrm{d} \Gamma_{\text {free }}}{\mathrm{d} x}=\frac{G_{F}^{2} m_{\mu}^{5}}{192 \pi^{3}} x^{2}\left(6-4 x+\frac{\alpha}{\pi} f(x)\right) \\
& x=\frac{2 E_{e}}{m_{\mu}} \quad 0<x \leq 1,
\end{aligned}
$$

where $\alpha=1 / 137.035999173(35)$ [4], $G_{F}$ is the Fermi constant, and $f(x)$ represents rather large, complicated radiative corrections that can significantly modify the electron spectrum. The function $f(x)$ is explicitly given by

$$
\begin{aligned}
f(x)= & {\left[\frac{5}{3 x^{2}}+\frac{16 x}{3}+\frac{4}{x}+(12-8 x) \ln \left(\frac{1}{x}-1\right)-8\right] } \\
& \times \ln \left(\frac{m_{\mu}}{m_{e}}\right)+(6-4 x)\left[2 \operatorname{Li}_{2}(x)-2 \ln ^{2}(x)+\ln (x)\right. \\
& \left.+\ln (1-x)\left(3 \ln (x)-\frac{1}{x}-1\right)-\frac{\pi^{2}}{3}-2\right] \\
& +\frac{(1-x)\left[34 x^{2}+\left(5-34 x^{2}+17 x\right) \ln (x)-22 x\right]}{3 x^{2}} \\
& +6(1-x) \ln (x) .
\end{aligned}
$$

The first term in the equation above is enhanced by the large logarithm $\ln \left(\frac{m_{\mu}}{m_{e}}\right)$. These large corrections vanish when integrated over the electron energy, as expected,

\footnotetext{
* Present address: Institute for Theoretical Particle Physics, KIT, D-76128 Karlsruhe, Germany.

† Present address: Albert Einstein Center for Fundamental Physics, Institut für Theoretische Physik, Universität Bern, Sidlerstrasse 5, CH-3012 Bern, Switzerland
}

due to the Kinoshita-Lee-Nauenberg (KLN) theorem [5, 6].

The experimental lifetime of a $\mu^{+}$stopped in matter, $\tau_{\mu}=2.1969803(22) \times 10^{-6} \mathrm{~s}$ (the most precise lifetime measurement for any unstable state [7]) determines the strength of weak interactions quantified by $G_{F}=1.1663788(7) \times 10^{-5} \mathrm{GeV}^{-2}$. Comparing it with the fine-structure constant and other high-precision electroweak observables, led to predictions for the top-quark and Higgs-scalar masses, before their discovery.

What happens when a $\mu^{-}$, rather than a $\mu^{+}$, is slowed down in matter? In vacuum the $\mu^{+}$and $\mu^{-}$lifetimes must be the same [8]; but in matter, their decays can appear quite different. As the $\mu^{-}$loses energy and starts to come to rest, it gets bound to nuclei of charge $Z$ due to their attractive Coulomb potential. The $\mu^{-}$quickly cascades down to the lowest $1 \mathrm{~S}$ atomic orbital, where it remains in a quantum wave function with a momentum distribution for which its average velocity is $\langle\beta\rangle \simeq Z \alpha$. The decreased energy of the bound muon causes the decay-inorbit (DIO) rate to slow down. In addition, the electron produced in the decay feels the same binding interaction, which increases its wave function near the decay region, and thus the decay probability. Interestingly, these two effects approximately cancel $[9,10]$ due to electromagnetic gauge invariance, and the difference between the overall decay rates of free and bound $\mu^{-}$is mainly due to the time dilation resulting from the bound muon's motion. (In matter, a $\mu^{-}$can also undergo capture, $\mu p \rightarrow \nu_{\mu} n$, which changes its effective lifetime [11, 12]. We do not discuss that process here.)

While Coulombic interactions with the nucleus do not significantly modify the overall DIO rate (about a $0.5 \%$ reduction from time dilation), they do make important changes to the spectrum of decay electrons.

As a result of the muon's velocity distribution, the spectrum in Eq. (1) is Doppler shifted and smeared. These effects render the radiative corrections embodied in $f(x)$ quite complicated. Although, as mentioned before, radiative corrections to the electron energy spectrum of a free muon decay are completely known up to second order in perturbation theory [2,3], no analysis of radiative corrections for a bound muon decay has been performed 
up to now. Methods traditionally used to calculate the DIO spectrum can not be simply extended to allow for the inclusion of radiative corrections.

In addition to the above effects, nuclear-recoil on DIO leads to a very small high-energy tail in the spectrum, extending all the way to electron energies $E_{e} \sim m_{\mu}$, well above the $E_{e} \sim m_{\mu} / 2$ end-point energy of free muon decay at rest. Although tiny, the DIO events near $E_{e} \sim m_{\mu}$ are an important background to searches for coherent $\mu-e$ conversion experiments that will probe for new exotic interactions at $10^{-16}$ sensitivity, four orders of magnitude beyond current bounds [13, 14]. A precise understanding of DIO, not only the very-high-energy tail, but the entire electron spectrum, will be important for calibrating and fully exploiting the intended sensitivity of those experiments.

Leading-order, i.e. excluding radiative corrections, theoretical predictions for the muon DIO spectrum, properly incorporating the effects of the Coulomb field of the nucleus on the muon decay, as well as the finite nuclear size, have been known for some time [15-17]. TWIST, an experimental muon decay program at TRIUMF, has provided the first precision test of those expectations for a wide range of DIO electrons with energies 18-70 MeV [18] using an aluminium stopping target. Although general agreement was found between the TWIST measurements [18] and theory [15-17], significant deviations were observed throughout the examined spectrum, particularly in the region around the free decay end point $\left(E_{e} \sim m_{\mu} / 2 \sim 52 \mathrm{MeV}\right)$ and at low energies 18-25 MeV.

As mentioned above, quantum electrodynamics (QED) corrections were ignored in Refs. [15-17]. Remarkably, the TWIST measurements seem to be precise enough to be sensitive to these subtle effects. Indeed, the TWIST Collaboration noted the need for but lack of suitable radiative corrections for their analysis. In this paper we provide a proper computation of radiative corrections for DIO in the aforementioned energy regions.

Why has it taken five years since the completion of the TWIST experiment for theory to catch up? The challenge is in evaluating radiative effects for bound particles, whose interaction with the nucleus cannot be treated as a perturbation.

However, a similar problem has been solved in quantum chromodynamics (QCD), in the context of heavyquark decays, already 20 years ago. Interestingly, it was noted that the necessary theoretical framework had existed in yet another area, the formalism of deep-inelastic lepton scattering on nuclei; in his 1995 lectures Shifman wrote "I see absolutely no reasons why the corresponding theory was worked out only recently and not 20 years ago" [19].

Our goal in this paper is to complete this cycle of theoretical developments by applying the main ideas to what should be a simpler case, namely QED. Toward that end, we derive a shape function that can be convoluted with the radiatively corrected free decay spectrum to approximate the effects of atomic binding. The range of va- lidity for that prescription should extend from roughly $m_{\mu} / 2$ (the free muon decay end point [1]) down to much lower energies, regions where spectral discrepancies have been uncovered by the TWIST Collaboration. Explaining those differences was, indeed, a major motivation for this work. Events with higher energy, resulting from nuclear-recoil effects are very rare, but extremely important near the DIO end point $\sim m_{\mu}$ where they are a background to searches for "new physics" via coherent $\mu-e$ conversion in atoms [20]. Incorporating radiative corrections in that region is not covered by our new method and is beyond the scope of this paper.

Following Schwinger's approach [21] to bound states, we calculate the muon-energy shift due to the field of the nucleus as an average value of the mass operator in the 1S state. The optical theorem relates its imaginary part to the muon decay rate. Denoting the sum of momenta of the neutrinos by $q$ we have

$$
\mathrm{d} \Gamma=\frac{G_{F}^{2}}{E_{1 S}} \operatorname{Im}\left(T_{\alpha \beta}\right) W^{\alpha \beta} \frac{\mathrm{d}^{4} q}{(2 \pi)^{3}},
$$

where $W^{\alpha \beta}$ is the neutrino tensor, and where we can formally write the charged particle tensor as (we use Schwinger's notation [21] and neglect the electron mass)

$$
T^{\alpha \beta}=\left\langle 1 \mathrm{~S}\left|\gamma^{\alpha} \frac{1}{\not 1-\not 1} \gamma^{\beta}\right| 1 \mathrm{~S}\right\rangle .
$$

We treat the nucleus as a static source of the electric field. Recoil-energy effects can be neglected for the range of electron energies considered here, since the recoil energy is $\delta E_{\mathrm{rec}} \sim \frac{m_{\mu}^{2}(Z \alpha)^{2}}{2 m_{N}}$, with $m_{N}$ denoting the nucleus mass. (In the high-energy region of the spectrum, recoilenergy effects are not suppressed by $(Z \alpha)^{2}$, modify the maximum allowed electron energy, and cannot be neglected [20].)

The Dirac wave function, describing the $1 \mathrm{~S}$ state of the muon, can be approximated in the leading $Z \alpha$ order by its large components [22]. To separate the muon motion inside the atom from the motion of the whole system, we rewrite the covariant derivative as $\Pi=m_{\mu} v+\pi$, where $v$ is the four-velocity of the muonic atom ( $v$ is timelike and $v^{2}=1$ ) and $\pi$ describes the residual motion of the bound muon; spatial components of $\pi$ are of order $m_{\mu} Z \alpha$, and $\left[\pi^{\alpha}, \pi^{\beta}\right]=i e F^{\alpha \beta}$. We now expand the spectrum in the region where $Q^{2}=\left(m_{\mu} v-q\right)^{2} \approx m_{\mu}^{2} Z \alpha$ (in the decay of a free muon, $Q$ would be the four-momentum of the electron; this condition requires the produced electron to be almost on-shell). Keeping only the leading corrections in $Z \alpha$ we get

$$
T^{\alpha \beta}=\left\langle 1 \mathrm{~S}\left|\gamma^{\alpha} \frac{\not}{Q^{2}+2 \pi \cdot Q} \gamma^{\beta}\right| 1 \mathrm{~S}\right\rangle .
$$

We exploit the lightness of the electron and decompose $Q$ using a lightlike vector $n, Q=v \cdot Q n+\delta Q$ with $n^{2}=$ $0, n \cdot v=1$ [23]. As long as $E_{e} \gg m_{\mu} Z \alpha$, we can neglect 
the term $\pi \cdot \delta Q$,

$$
\begin{aligned}
& \frac{1}{\pi} \operatorname{Im}\left(T^{\alpha \beta}\right)=\frac{m_{\mu}}{2} \operatorname{Tr}\left[\gamma^{\alpha} \not \mathcal{Q} \gamma^{\beta}(1+\psi)\right] \\
& \times \int \mathrm{d} \lambda s(\lambda) \delta\left(Q^{2}+2 \lambda v \cdot Q\right),
\end{aligned}
$$

where $s(\lambda)$ is a QED analog of the shape function [23$26]$ that in our case can be explicitly evaluated using the muon's Schrödinger wave function $\psi(x)$,

$$
s(\lambda)=\int \mathrm{d}^{3} x \psi^{\star}(x) \delta(\lambda-n \cdot \pi) \psi(x) .
$$

Great simplification can be achieved through a judicious choice of the electromagnetic gauge, reducing the effect of the Coulomb interaction on the electron. In the light-cone gauge, $n \cdot A=0$, we have

$$
s(\lambda)=\int \frac{\mathrm{d}^{3} k}{(2 \pi)^{3}} \psi_{g}^{\star}(\vec{k}) \delta(\lambda+\vec{n} \cdot \vec{k}) \psi_{g}(\vec{k}),
$$

where $\psi_{g}(\vec{k})$ is the muon wave function in momentum space calculated in the light-cone gauge. Neglecting terms quadratic in the smearing variable $\lambda$, the delta function in Eq. (6), describing the electron's on-shell condition, can be rewritten as

$$
\delta\left(Q^{2}+2 \lambda v \cdot Q\right) \simeq \delta\left(q^{2}-\tilde{m}^{2}+2 \tilde{m} \tilde{E}\right),
$$

with $\tilde{E}=E_{e}+\lambda+\frac{(Z \alpha)^{2} m_{\mu}}{2}$ and $\tilde{m}=m_{\mu}+\lambda$. Note that in the free muon decay, the on-shell condition for the electron is $q^{2}-m_{\mu}^{2}+2 m_{\mu} E_{e}=0$. The muon mass and the electron energy can be replaced by $\tilde{m}$ and $\tilde{E}$ also in the matrix element in front of the delta function, since this introduces a change of higher order in $Z \alpha$, beyond our target accuracy.

Within that accuracy, the radiative corrections can be included by substituting a matrix element squared including virtual and real radiation for the tree-level expression in front of the integral in Eq. (6). As a result, the expression for the DIO spectrum becomes a convolution of the shape function with the spectrum of the freemuon decay, in a form familiar from heavy-quark physics [27],

$$
\frac{\mathrm{d} \Gamma}{\mathrm{d} E_{e}}=\left.\int \mathrm{d} \lambda s(\lambda) \frac{\mathrm{d} \Gamma_{\text {free }}}{\mathrm{d} z} \frac{\mathrm{d} z}{\mathrm{~d} E_{e}}\right|_{z \rightarrow z(\lambda)},
$$

where $\frac{d \Gamma_{\text {free }}}{d z}$ denotes the differential decay rate of a free muon, including radiative corrections, with a daughter electron carrying energy $E_{e}=z m_{\mu} / 2$, and

$$
z(\lambda)=\frac{2\left(E_{e}+\lambda\right)+(Z \alpha)^{2} m_{\mu}}{m_{\mu}+\lambda} .
$$

Note that we have kept a term quadratic in $Z \alpha$, arising from the binding energy of the muon, $E_{1 \mathrm{~S}} \approx$ $m_{\mu}\left(1-\frac{(Z \alpha)^{2}}{2}\right)$. This term shifts the spectrum, since the maximum energy of the electron is $E_{1 \mathrm{~S}}$ rather than $m_{\mu}$. Around $m_{\mu} / 2$, the derivative of the spectrum with respect to the energy behaves like $\frac{1}{Z \alpha}$, so we need to have the quadratic term in order to obtain the result correct to $\mathcal{O}(Z \alpha)$.

Eq. (10) is noteworthy in several respects. First, the final state characterized by the observed value of $E_{e}$ arises from a superposition of contributions: the energy of the electron is modified by the motion of the muon and by the decay electron's interaction with the nuclear field. The probability of observing $E_{e}$ should involve a square of the sum of probability amplitudes; but the leading binding correction results in the sum of probabilities.

Second, in our present QED analysis, the shape function is derived from first principles. This is in contrast to QCD, where it was introduced [23-26]. There, because of strong interactions, the shape function cannot be computed. Instead it has to be modelled, and constrained from experimental data.

Finally, the decay spectrum $\mathrm{d} \Gamma_{\text {free }} / \mathrm{d} z$ refers to a free electron, although we know that its interaction with the nucleus must be accounted for. Information about this interaction is encoded in $s(\lambda)$. This is possible thanks to gauge invariance. The light-cone gauge enables us to approximately treat the electron as a free particle.

As previously remarked, our analysis closely resembles, and uses the techniques employed for, the studies of heavy-quark decays. Separation of disparate physical scales is at the basis of the heavy-quark expansions employed there. Therefore, it is worth noticing the corresponding energy scales which need to be taken into account when considering muon DIO. As is manifest from the derivations presented above, the essential idea is, like in heavy-quark systems, the separation of bound-state energy scales from a hard energy scale, given by $m_{\mu}$. The typical bound-state momentum in a muonic atom is given by $m_{\mu} Z \alpha$. Therefore, the expansion parameter in our computation is given by $\left(m_{\mu} Z \alpha\right) / m_{\mu}=Z \alpha$, which plays a role analogous to $\Lambda_{Q C D} / m_{Q}$ in heavy quark effective theory (where $\Lambda_{Q C D}$ is the QCD scale, and $m_{Q}$ the heavy-quark mass). However, this separation of boundstate effects from the hard scale is no longer possible in the high-energy region of the DIO spectrum, $E_{e} \sim m_{\mu}$. In this region, in order to produce an on-shell electron in the final state, hard photons need to be exchanged between the muon (or the electron) and the nucleus. Our formalism is therefore expected to work in the energy region $E_{e}<\left(m_{\mu} / 2\right)+m_{\mu} Z \alpha$. A proper treatment of the higher-energy part of the electron spectrum is beyond the scope of this work. Note also that the convolution formula Eq. (10) allows us to calculate only the dominant corrections; i.e., it does not include all corrections of or$\operatorname{der}(Z \alpha)^{2}$, and beyond. These sub-leading effects would appear as additional, parametrically suppressed, shape functions. Subleading shape functions have been investigated to some extent in the context of heavy-quark decays, see e.g. Ref. [28], and their study is quite involved. The difficulty in treating and estimating the size of these 
subleading effects in heavy-quark systems is mainly due to the fact that shape functions encode nonperturbative effects in the QCD case. In our QED case the shape function can be derived from first principles, and it is easier to estimate the size of the neglected subleading terms. To do that, we can (i) recompute the shape function by extracting it from a (fictitious) two-body bound decay of the muon, which, without considering radiative corrections, can be computed exactly along the lines of Ref. [29]. This provides a shape function which coincides with our previous computation at order $Z \alpha$ but contains different higher-order terms. (ii) Compare the muon DIO spectrum computed exactly but without radiative corrections, i.e. along the lines of Ref. [20], with the spectrum computed with our convolution formula using the Bornlevel free decay rate. The difference between the two spectra is due to neglected $\mathcal{O}\left((Z \alpha)^{2}\right)$ terms in our shape function. We have performed both checks, and found that indeed the effect of higher-order terms is always of order $(Z \alpha)^{2} / 2$, and is never larger than $1 \%$. This explicitly shows that we can safely neglect subleading shape functions to describe the available experimental data. The complete order $(Z \alpha)^{2}$ corrections to DIO can, in principle, be incorporated by combining the more exact Coulombic treatment in Ref. [20] with the shape function approach to ordinary radiative corrections described in this work. The added effect is expected to be relatively small for $\mathrm{Al}$ with $\mathrm{Z}=13$, but could become important for much larger $\mathrm{Z}$.

We also mention that finite-nuclear-size effects are more important in muonic atoms than in usual electronic atoms. The muon, because of its relatively large mass, spends more of its time close to the nucleus. We take into account the finite size of the nucleus when comparing our results with experimental data, and calculate the muon wave function numerically for an assumed model of charge distribution inside the nucleus. More concretely, we calculate the muon wave function as in Refs. [20, 29, 30], using a two-parameter Fermi charge distribution:

$$
\varrho(r)=\frac{\varrho_{0}}{1+e^{\frac{r-r_{0}}{a}}} .
$$

In the numerical evaluation we have focused on aluminium, $Z=13, r_{0}=2.84 \mathrm{fm}$ and $a=0.569 \mathrm{fm} \mathrm{[31],} \mathrm{the}$ target used in the TWIST experiment [18] and considered as the muon stopping material for the $\mu-e$ conversion searches at Fermilab and J-PARC [13, 14]. Including binding energy, $E_{1 \mathrm{~S}}^{\mathrm{Al}} \approx m_{\mu}-0.5 \mathrm{MeV}$. Fig. 1 shows the function $s(\lambda)$ calculated, using Eq. (8). As expected, the main support of this function comes from the region $\pm Z \alpha m_{\mu}$ around 0 , determined by the main support of the muon wave function in momentum space. We also note that uncertainties in the modeling of finite-nuclearsize effects were analyzed in Ref. [20]; they are not larger than our target accuracy in the present analysis, and can be safely neglected for our purposes here.

The normalization of the spectrum is very important when comparing theoretical calculations with data. The

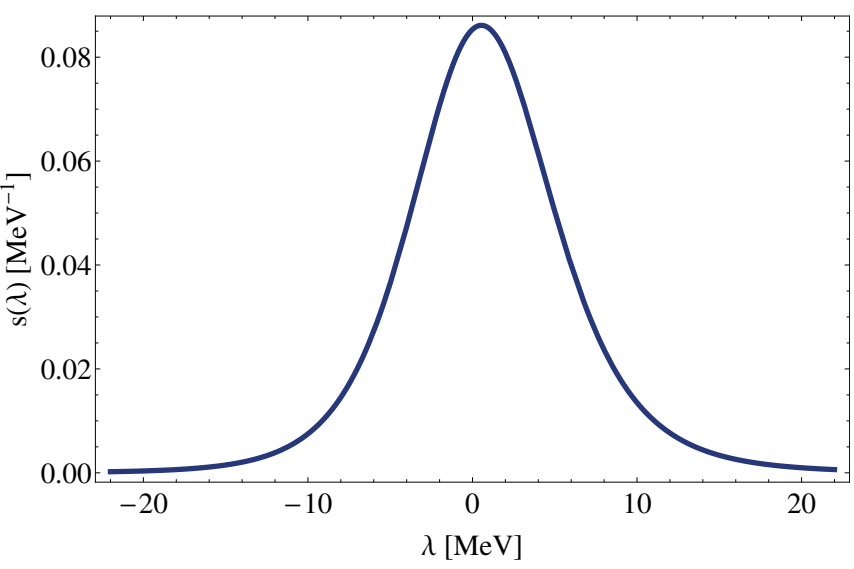

FIG. 1. The function $s(\lambda)$ calculated numerically for the aluminium nucleus. The half-width of the peak is approximately $Z \alpha m_{\mu} \simeq 10 \mathrm{MeV}$. The slight asymmetry reflects the final state interaction of the electron.

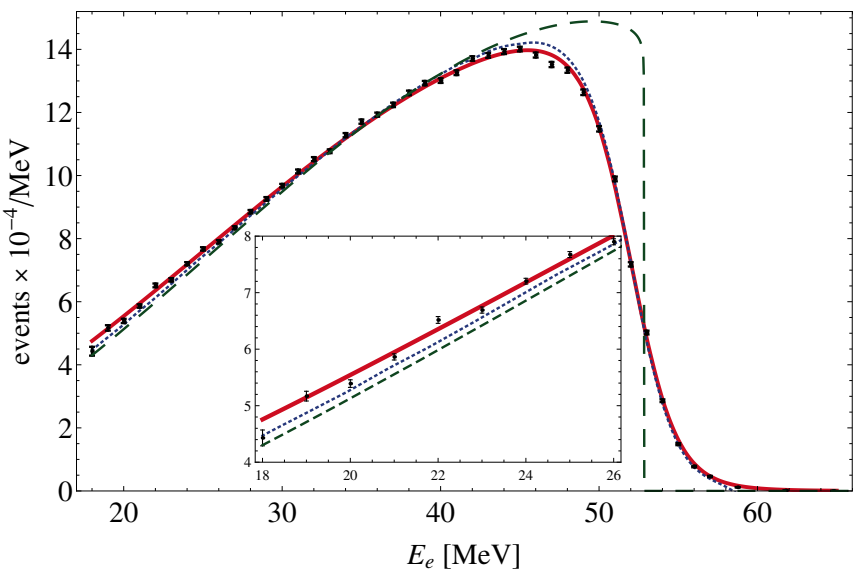

FIG. 2. Theoretical electron-energy spectra for muon DIO compared with TWIST data (black points) [18]. The solid red (dotted blue) line is the spectrum with (without, [17, 20]) $\mathcal{O}(\alpha)$ radiative corrections. The green dashed line represents the spectrum of the free muon decay with radiative corrections [32]. Near the free muon end point, $52.8 \mathrm{MeV}$, there is a large negative QED correction which pushes the dashed line to zero, and the solid line below the dotted one. In the lowenergy region, magnified in the inset, both radiative (dashed) and binding (dotted) corrections are positive, leading to an increase of low-energy electrons. The solid line includes both effects.

TWIST data, in addition to statistical errors, also has an energy scale uncertainty of $\pm 0.2 \%$. We include it to improve the agreement between data and our calculation by expressing the spectrum as a function $p$ of two fit parameters $N$ and $a$,

$$
p(N, a)=N \frac{\mathrm{d} \Gamma(a \zeta)}{\mathrm{d} \zeta} .
$$

The parameter $a$ accounts for both experimental and theoretical energy scale uncertainties. Its fitted value, 
$a \approx 1.0015$, differs from unity within the error range claimed by TWIST, i.e. $\pm 2 \times 10^{-3}$.

Figure 2 compares TWIST experimental data, obtained from the decay of muons bound in aluminum with theoretical spectra (free, lowest-order bound and including radiative corrections). We see that radiative corrections [obtained via Eq. (10)] bring theory and experiment into good agreement. The improvement is further demonstrated in Fig. 3 which highlights the difference between theory and experiment, with and without radiative corrections. The rather sizable radiative corrections (as large as 6\%) rearrange the spectrum, but tend to cancel in the total decay rate. We note that improved agreement for $E_{e} \simeq 52-54 \mathrm{MeV}$ is due in part to a 0.15 per cent scale shift in $a$ [see Eq. (13)] when our normalized fit to data includes radiative corrections. Also, the plot does not address experimental points above $54 \mathrm{MeV}$, where our approximations may start to fail.

For the convenient use of our results, we provide a simple fit to the spectrum which should be accurate up to effects of order $(Z \alpha)^{2} \simeq 0.01$ for aluminium. Introducing a dimensionless variable $\zeta=\frac{2 E_{e}}{E_{1 \mathrm{~S}}}$ we find

$$
\frac{1}{\Gamma} \frac{\mathrm{d} \Gamma}{\mathrm{d} \zeta} \approx \begin{cases}0.076+0.024 \zeta+5.92 \zeta^{2}-4.16 \zeta^{3} & 0.4<\zeta<0.76 \\ 7.12 \zeta^{2}-5.15 \zeta+0.966 \ln (1-\zeta)+2.87 & 0.76<\zeta<0.92 \\ (0.085 \zeta+1.24) /(0.714+\exp [36.7(\zeta-1)]) & 0.92<\zeta<1.05\end{cases}
$$

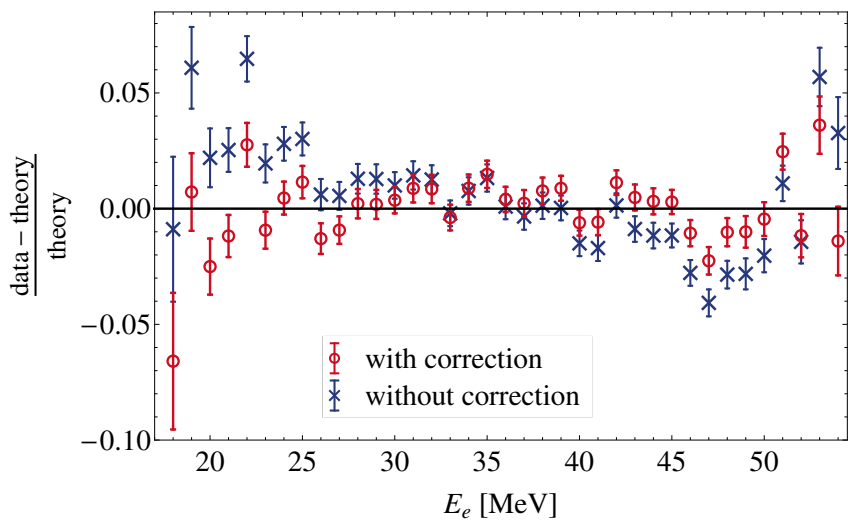

FIG. 3. Relative difference between data and the theory prediction with (circles) and without (crosses) radiative corrections. The difference between the measured and the calculated spectrum is normalized to the theoretical spectrum, appropriate for each case.

The fit is normalized such that $\Gamma=\int_{0}^{2} \frac{d}{d \zeta}$. The highenergy spectrum, including recoil and binding effects is given in Ref. [20]; however, radiative corrections in that region have not been included.

In Fig. 4, the solid line describes the $\mathcal{O}(\alpha)$ corrections to the lowest order DIO spectrum for a bound muon as a function of $\zeta$. For comparison, the free muon radiative corrections are given as a function of $x$ (see Eq.(1)). In the lower energy region, accessible to both bound and free decays, the radiative corrections are similar for the two cases, with a part of the shift coming from a difference in the $\zeta$ and $x$ variables. Differences are largest, close to the free muon decay spectrum end point, where a logarithmic enhancement in the free case $(\ln (1-x)$ singularity) is smeared for the DIO case, as illustrated by the solid curve.

In summary, we have derived a new method for approximating QED radiative corrections to muon DIO

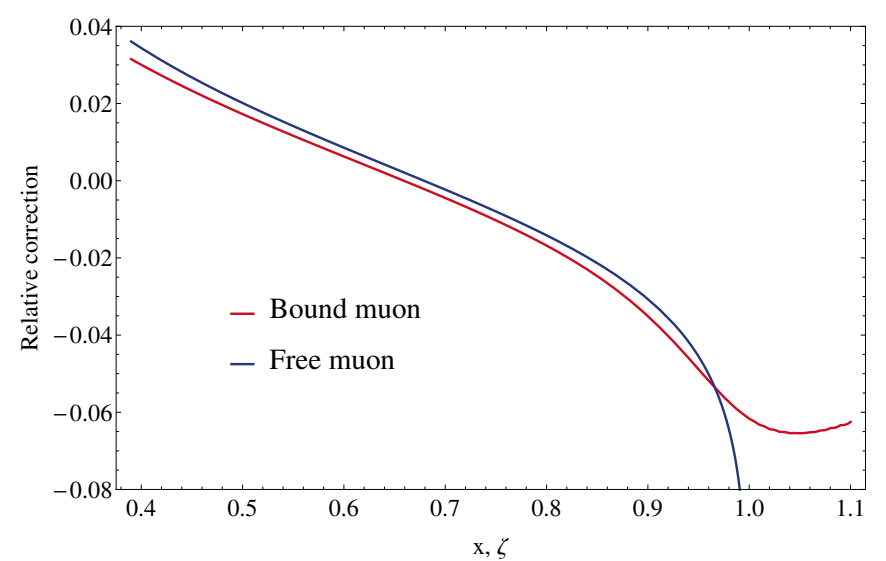

FIG. 4. Relative leading $\mathcal{O}(\alpha)$ radiative corrections to the electron spectrum for bound and free muons. The solid (dashed) line corresponds to the corrections for a bound (free) muon as a function of the electron energy variable $\zeta(x)$.

rates based on a formalism developed for heavy-quark weak decays in QCD. Its general features are in good accord with expectations based on Lorenz and gauge invariance. The radiative corrections are quite large near the spectral peak and at low energies, regions where the TWIST experiment had discovered discrepancies with theory. As a result, our new improved theoretical spectrum is now in excellent quantitative agreement with experiment and, where applicable, can be confidently used in future searches for exotic new physics.

\section{ACKNOWLEDGMENTS}

A.C., M.D., X.G.T. and R.S. were supported by Science and Engineering Research Canada (NSERC). W.J.M. was supported by the United States DOE un- 
der Grant No. DE-ACO2-98CH10886.

[1] L. Michel, Proc. Phys. Soc. A 63, 514 (1950).

[2] T. Kinoshita and A. Sirlin, Phys. Rev. 113, 1652 (1959).

[3] C. Anastasiou, K. Melnikov, and F. Petriello, J. High Energy Phys. 09, (2007) 014.

[4] R. Bouchendira et al., Ann. Phys. (N.Y.) 525, 484 (2013).

[5] T. Kinoshita, J. Math. Phys. 3, 650 (1962).

[6] T. D. Lee and M. Nauenberg, Phys. Rev. 133, 1549 (1964).

[7] D. Webber et al., Phys. Rev. Lett. 106, 041803 (2011).

[8] R. Streater and A. Wightman, PCT, Spin and Statistics, and All That (Princeton University Press, Princeton, NJ, 2000).

[9] H. Überall, Phys. Rev. 119, 365 (1960).

[10] A. Czarnecki, G. P. Lepage, and W. J. Marciano, Phys. Rev. D 61, 073001 (2000).

[11] V. Andreev et al., Phys.Rev.Lett. 110, 012504 (2013).

[12] A. Czarnecki, W. J. Marciano, and A. Sirlin, Phys. Rev. Lett. 99, 032003 (2007).

[13] G. Onorato, Nucl. Instrum. Meth. A718, 102 (2013).

[14] Y. Kuno, PTEP 2013, $022 \mathrm{C01}$ (2013).

[15] P. Hänggi, R. Viollier, U. Raff, and K. Alder, Phys. Lett. 51B, 119 (1974).

[16] R. Watanabe, M. Fukui, H. Ohtsubo, and M. Morita, Prog. Theor. Phys. 78, 114 (1987).

[17] R. Watanabe et al., At. Data and Nucl. Data Tables 54, 165 (1993).
[18] A. Grossheim et al., Phys. Rev. D 80, 052012 (2009).

[19] M. A. Shifman, in ITEP Lectures on Particle Physics and Field Theory, edited by M. A. Shifman (World Scientific, Singapore, 1999), hep-ph/9510377.

[20] A. Czarnecki, X. Garcia i Tormo, and W. J. Marciano, Phys. Rev. D 84, 013006 (2011).

[21] J. Schwinger, Particles, Sources and Fields (AddisonWesley, Reading, MA, 1973), Vol. 2.

[22] R. Szafron, Acta Phys. Polon. B 44, 2289 (2013).

[23] T. Mannel and M. Neubert, Phys. Rev. D 50, 2037 (1994).

[24] M. Neubert, Phys. Rev. D 49, 3392 (1994).

[25] M. Neubert, Phys. Rev. D 49, 4623 (1994).

[26] I. I. Y. Bigi, M. A. Shifman, N. G. Uraltsev, and A. I. Vainshtein, Int. J. Mod. Phys. A9, 2467 (1994).

[27] F. De Fazio and M. Neubert, J. High Energy Phys. 06, (1999) 017.

[28] S. W. Bosch, M. Neubert and G. Paz, J. High Energy Phys. 11 (2004) 073.

[29] X. Garcia i Tormo, D. Bryman, A. Czarnecki, and M. Dowling, Phys. Rev. D 84, 113010 (2011).

[30] A. Czarnecki, W. J. Marciano, and K. Melnikov, in Physics at the First Muon Collider, edited by S. Geer and R. Raja (AIP, Woodbury, 1998), p. 409, hep-ph/9801218.

[31] H. de Vries et al., At. Data Nucl. Data Tables 36, 495 (1987).

[32] D. Atwood and W. J. Marciano, Phys. Rev. D 41, 1736 (1990). 